\title{
Keeping the lid on herpes simplex virus
}

Herpes simplex virus 1 (HSV-1) establishes a lifelong association with infected hosts, often causing severe disease when the host is immunocompromised. In a recent issue of Science, Knickelbein and colleagues report the identification of part of the mechanism that the host uses to keep HSV-1 under control.

HSV-1 persists inside neurons in a latent state that is maintained by viral and neuronal factors. Decreased immune function can lead to the reactivation of HSV-1 and disease, including cold sores and ocular lesions. It was already known that $\mathrm{CD}^{+} \mathrm{T}$ cells associate with HSV-1infected neurons. Activated $\mathrm{CD}^{+}$ T cells secrete specialized secretory organelles, called lytic granules, into the immunological synapse between activated $\mathrm{T}$ cells and target cells. This leads to the apoptosis of most infected cells, but not latently infected neurons. Lytic granules contain perforin, which enables other lytic granule proteins, such as the serine protease granzyme B, to

.... T-cell response to virus-infected cells can quickly remove a protein that is important for the initiation of the lytic replication cycle. enter the target cell. Knickelbein and colleagues show that $\mathrm{CD} 8^{+} \mathrm{T}$ cells release lytic granules into the synapse adjacent to HSV-1-infected neurons. The importance of the proteins secreted by the $T$ cells in the control of HSV-1 was shown by an ex vivo assay in which $\mathrm{T}$ cells that lacked either granzyme B or perforin were less efficient than fully functional $\mathrm{T}$ cells in preventing HSV-1 from reactivating in neurons. The fact that perforin-deficient cells were more profoundly compromised than granzyme B-deficient cells suggested the involvement of other lytic granule components, such as granzyme $\mathrm{A}$, that also require perforin to enter cells.

Because granzyme B did not kill infected neurons, the authors tested whether granzyme B could control infection by cleaving essential viral proteins. Bioinformatics revealed two putative granzyme B cleavage sites in HSV-1 immediate early protein ICP4, which is required to switch transcription from latent to lytic genes. In a proteolysis assay, purified granzyme B cleaved ICP4 in crude cell lysates and, following purification by immunoprecipitation, in a time- and concentration-dependent manner.

The observed rapid cleavage of ICP4 indicates that a T-cell response to virus-infected cells can quickly remove a protein that is important for the initiation of the lytic replication cycle.

Based on these and previous results, the authors propose that the latency of HSV-1 is maintained by three factors: viral factors that reduce the synthesis of lytic phase proteins,

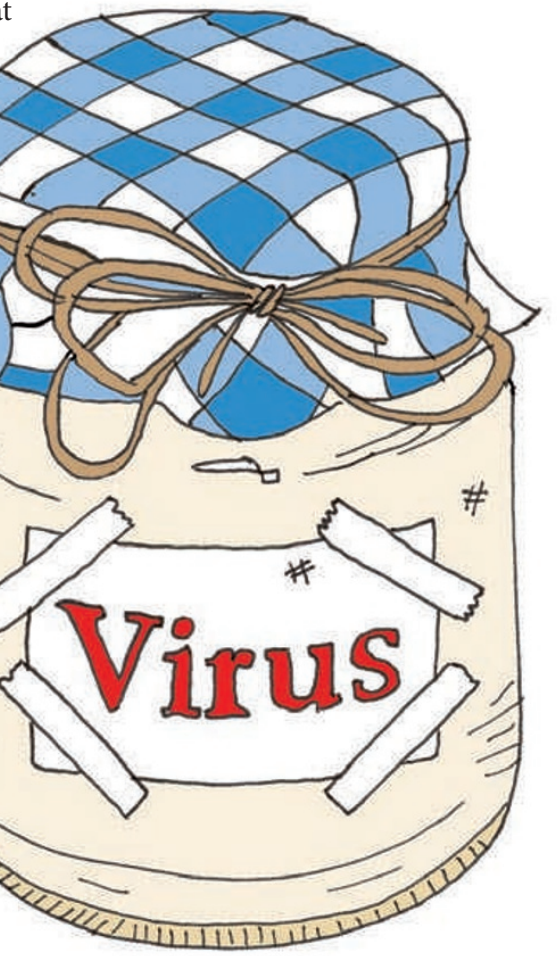

epigenetic control of viral gene expression and $\mathrm{CD} 8^{+} \mathrm{T}$-cell release of effector molecules, including interferon- $\gamma$ and granzyme B, that target ICP4. This delicate balance ensures that HSV-1 is maintained in a latent state that does not cause disease.

Christiaan van Ooij

ORIGINAL RESEARCH PAPER Knickelbein, J. E. et al. Noncytotoxic lytic granule-mediated CD8 ${ }^{+}$ T cell inhibition of HSV-1 reactivation from neuronal latency. Science 322, 268-271 (2008) 